\title{
FAULT MONITORING AND SIGNAL ANALYSIS OF AEROENGINE ROTOR VIBRATION
}

\author{
Shuang Xia ${ }^{1}$ \\ ${ }^{1}$ Sanya Aviation and Tourism College, Sanya, Hainan 572000, China \\ Email:sx8tsl@126.com
}

\begin{abstract}
In the process of aeroengine development and operation, fault monitoring is very important, especially for the core part, rotor. In this study, the Fourier separation method and support vector machine (SVM) were combined to realize the vibration monitoring of aeroengine rotor fault, and the independent component analysis (ICA) was used to improve the method for extracting vibration characteristics. After that, the two monitoring methods before and after the improvement were compared through experiment. The experimental results showed that the two feature extraction methods could extract fault vibration features from the original vibration signals, but the vibration features extracted by FDM could not effectively distinguish the fault vibration source, and ICA-FDM could effectively distinguish the fault vibration source after blind source separation; after training, SVM based on ICA-FDM could identify the single rotor outer ring fault of the simulation platform with higher accuracy and precision and lower false alarm rate than the FDM based SVM.
\end{abstract}

Keywords: Engine, Fault Monitoring, Vibration Characteristics, Rotor.

\section{Introduction}

In an aircraft, the engine [1] is its power source. In the process of operation, various parts of the engine will inevitably produce vibration due to the temperature, air pressure, rotation of rotating parts, etc. in the process of high-speed operation, and each part will have different vibration signals due to different materials, shapes, etc., that is to say, it has specific vibration characteristics [2]. The rotor is the core part of the aeroengine. The main vibration source of the whole engine and its components is caused by the rotation of rotor. For an engine, the most ideal state is that the rotor does not produce vibration when rotating, but it is impossible in the actual development and operation of the engine, because of the friction between components and the uneven distribution of rotor mass. With the continuous vibration, the rotor of the engine will gradually produce faults, so it is necessary to monitor the fault of the engine rotor in order to adjust the engine in time. Collecting vibration signals with sensors in the engine and analyzing its vibration characteristics can effectively monitor the state of aeroengine and obtain its fault type [3]. Ma et al. [4] established the dynamic model of the dualrotor-casing system by using the finite element method, verified the model, and found that the frequency components of different combinations in the frequency spectrum could be used as the diagnostic frequency of the dual-rotor aeroengine in the event of rub impact fault. Wang et al. [5] diagnosed the fault of the engine rotor using 1-D spectral analysis and tested the reliability of the diagnosis method using the actual experiment. Ding et al. [6] proposed a rotor fault diagnosis method based on ensemble empirical mode decomposition (EEMD) and neighborhood rough set (NRS) and verified the effectiveness of the method by simulation experiments. In this study, Fourier separation method was combined with support vector machine (SVM) to realize the vibration monitoring of aeroengine rotor fault, and the method of extracting vibration features was improved using independent component analysis (ICA). After that, the two monitoring methods before and after the improvement were compared.

\section{Feature Extraction of Vibration Signal of Aeroengine Fault Rotor}

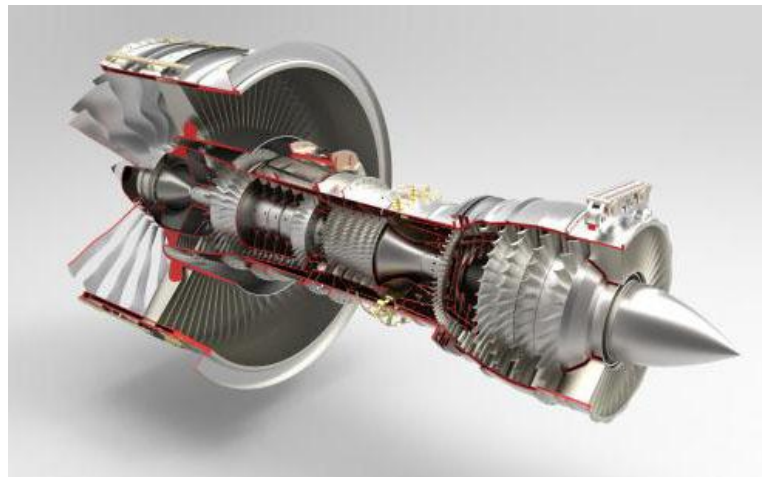

Figure 1: Structural diagram of aeroengine 
The original aeroengine is similar to the automobile engine, which uses the expansion thrust generated by the rapid combustion of fossil fuel in the confined space to push the piston to generate power; with the increasing demand for the flight speed of the aircraft, the turbojet engine appears, and its structure is shown in Figure 1, including the air inlet, compressor, combustion chamber, turbine and tail nozzle, in which the turbine is the main part of the whole jet engine. The structure of the turbine mainly includes rotating shaft, rotor and the working blades connected to the rotor.

In the process of rotor rotation, the vibration source will be formed to drive other parts to vibrate, so that the engine vibration signal collected by the vibration sensor is an aliasing signal, including meaningless noise signal. The required fault signal either overlaps with other vibration signals or is covered by noise background, so that the effective fault information can not be obtained directly from the signal collected by the sensor.

\subsection{Feature extraction based on Fourier transform}

Fourier decomposition method (FDM) is an algorithm to decompose signals by using Fourier transform [7].
In this study, the vibration signals collected by sensors are expressed as follows:

$$
x(t)=\sum_{i=1}^{M} y_{i}(t)+r(t)
$$

where $x(t)$ is the vibration signal collected by the sensor, $y_{i}(t)$ is the i-th vibration sub-signal (the sub-signals are zero mean functions, they are orthogonal to each other, and the envelope amplitude and instantaneous frequency in the analytical function of the sub-signal $\geq 0$ ), $M$ indicates the number of sub-signals, and $r(t)$ is the residual component. As the vibration of aeroengine is mechanical vibration and the mechanical vibration is often nonlinear and nonstationary in a certain period of time, it is supposed that the vibration signal $x(t)$ collected has dirichlet condition in time $\left[t_{1}, t_{1}+T_{0}\right]$ [8], then $x(t)$ can be expressed as:

$$
\left\{\begin{array}{l}
x(t)=x_{T 0}(t) \omega(t) \\
\omega(t)=1 \quad t \in\left[t_{1}, t_{1}+T_{0}\right] \\
\omega(t)=0 \quad \text { other }
\end{array}\right.
$$

$x_{T 0}(t)$ in equation (2) is processed by Fourier expansion, then there is:

$$
\left\{\begin{array}{l}
x_{T \mathrm{O}}(t)=a_{\mathrm{O}}+\sum_{k=1}^{\infty}\left(a_{k} \cos \left(k \omega_{\mathrm{O}} t\right)+b_{k} \sin \left(k \omega_{\mathrm{O}} t\right)\right) \\
a_{\mathrm{O}}=\int_{t_{1}}^{t_{1}+T_{\mathrm{O}}} x_{T \mathrm{O}}(t) d t \\
a_{k}=\frac{2 \int_{t_{1}}^{t_{1}+T_{\mathrm{O}}} x_{T \mathrm{O}}(t) \cos \left(k \omega_{\mathrm{O}} t\right) d t}{T_{\mathrm{O}}} \\
b_{k}=\frac{2 \int_{t_{1}}^{t_{1}+T_{\mathrm{O}}} x_{T \mathrm{O}}(t) \sin \left(k \omega_{\mathrm{O}} t\right) d t}{T_{\mathrm{O}}} \\
\omega_{\mathrm{O}}=2 \pi / T_{\mathrm{O}}
\end{array}\right.
$$

After transforming equation (3), there is:

$$
\left\{\begin{array}{l}
x_{T 0}(t)=a_{0}+\operatorname{Re}\left(z_{T 0}(t)\right)=a_{0}+\sum_{k=1}^{\infty}\left(c_{k} \cos \left(-j k \omega_{0} t\right)+c_{k}^{*} \sin \left(-j k \omega_{0} t\right)\right) \\
c_{k}=a_{k}-j b_{k} \\
c_{k}^{*}=a_{k}+j b_{k}
\end{array}\right.
$$

The analytic Fourier intrinsic band functions (AFIBFs) of $x(t)$ can be obtained from equation (4):

$$
z_{T 0}(t)=\sum_{k=1}^{M} a_{i}(t) \exp \left(j \varphi_{i}(t)\right)
$$

The vibration signal sequence collected by the sensor is expanded to a limited number of AFIBFs using equation (5), from which the vibration characteristics of different components are obtained, i.e. frequency and envelope amplitude [9].

\subsection{Blind source separation based on ICA}

In the process of decomposing the vibration signals to get AFIBFs of different components with FDM, the vibration sources of the mixed vibration 
signals are separated, such as the background noise of vibration. Although FDM can decompose the mixed vibration signal simply and stably, which is suitable for the condition monitoring of aeroengine, this method can only get characteristics of the basic frequency and double frequency of the engine rotor vibration and can not distinguish the non-basic frequency and non-double frequency vibration sources, which will affect the subsequent fault monitoring. In order to solve this problem, the separation of aliasing signal is realized by ICA [10].

The aliasing signal of engine can be expressed as:

$$
\left\{\begin{array}{l}
\mathbf{x}=\mathbf{H} \bullet \mathbf{s} \\
\mathbf{x}=\left(x_{1}, x_{2}, \Lambda, x_{n}\right)^{\mathrm{T}} \\
\mathbf{s}=\left(s_{1}, s_{2}, \Lambda, s_{n}\right)^{\mathrm{T}}
\end{array}\right.
$$

where $\mathbf{x}$ is the set of observation signals, $\mathbf{S}$ is a set of independent source signals, and $\mathbf{H}$ is a mixed matrix.

In this study, the separation matrix which is 1 after multiplying with the mixed matrix is calculated using signal to noise ratio, and the formula is as follows:

$$
F(\mathbf{y})=10 \log \frac{\mathbf{y y}^{\mathrm{T}}}{(\tilde{\mathbf{y}}-\mathbf{y})(\tilde{\mathbf{y}}-\mathbf{y})^{\mathrm{T}}}
$$

where $F(\mathbf{y})$ is the signal-to-noise ratio between the separated signal and source signal and $\tilde{\mathbf{y}}$ is the moving average separation signal for replacing source signal s. As $\left\{\begin{array}{l}\mathbf{y}=\mathbf{W x} \\ \tilde{\mathbf{y}}=\mathbf{W} \tilde{\mathbf{x}}\end{array}\right.$, objective function (7) is transformed into:

$$
F(\mathbf{W}, \mathbf{x})=10 \log \frac{\mathbf{W} \mathbf{x} \mathbf{x}^{\mathrm{T}} \mathbf{W}^{\mathrm{T}}}{\mathbf{W}(\tilde{\mathbf{x}}-\mathbf{x})(\tilde{\mathbf{x}}-\mathbf{x})^{\mathrm{T}} \mathbf{W}^{\mathrm{T}}}
$$

Then the gradient of both sides of equation (8) is calculated using separation matrix $\mathbf{W}$, and the zero point of the gradient is calculated, so as to obtain the extreme point of objective function (8) and separation matrix $\mathbf{W}$.

After obtaining the separation matrix through the above steps, the collected aliasing signal is separated, and then the separated signal is decomposed by FDM to obtain the vibration characteristics and judge whether the engine rotor has fault.

\section{SVM}

The vibration feature extraction method of engine rotor is as shown above, but in the actual rotor fault monitoring, even if the fault vibration feature is obtained by feature extraction, the efficiency of artificial judgment is still relatively low, so a classification algorithm is needed to quickly determine whether the engine rotor is faulty according to the fault vibration feature.

SVM [12] is an intelligent algorithm that can learn by itself. Its basic principle is to project data into high-dimensional space using kernel function, then find the optimal classification boundary according to the linear division principle, and classify the detection data taking the classification boundary as the standard.

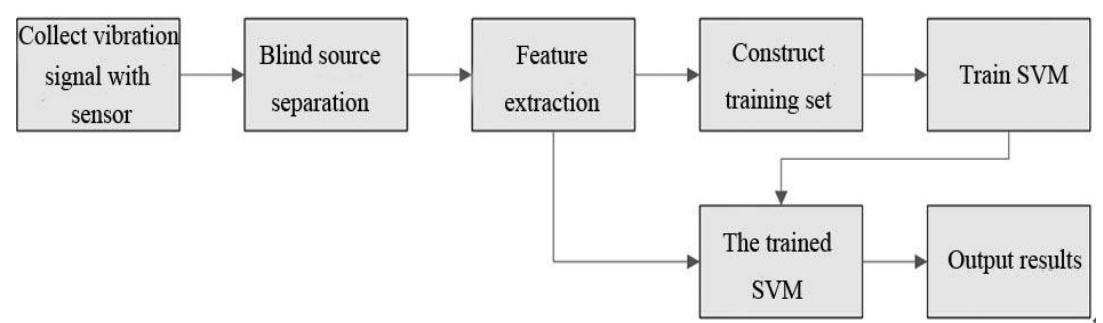

Figure 2: The flow of engine rotor fault monitoring based on SVM and engine vibration characteristics

The monitoring flow of aeroengine rotor failure is shown in Figure 2.

(1) Firstly, vibration signals of engine are collected by sensors during operation, including vibration signals in normal operation and failure states.

(2) The collected vibration signals are processed by blind source separation.

(3) Vibration features are extracted using FDM.

(4) The extracted vibration signal features are constructed as training sets and then input into SVM. Appropriate kernel functions and penalty parameters are selected. The decision function is obtained after calculation. The calculation formula of the decision function is:

$$
f(x)=\operatorname{sgn}\left(\sum_{i=1}^{l} a_{i} y_{i} K\left(x_{i}, x_{j}\right)+b\right)
$$

where $a$ stands for the set of $a_{i}, a_{i}$ is Lagrangian coefficient [14], $l$ stands for sample size, $K\left(x_{i}, x_{j}\right)$ is the kernel function, and $C$ is the penalty parameter.

SVM training finishes.

(5) When the fault of the engine rotor is monitored by the trained SVM, the first three steps are the same as (1) (2) (3): firstly, the vibration 
information is collected by the sensor, the vibration signal collected based on the maximum signal-tonoise ratio is processed by blind source separation, and features are extracted by FDM and input to the trained SVM to get the output result.

\section{Experimental Analysis 4.1 Experimental environment}

In this study, the self built simulation engine rotor vibration platform was used to simulate the vibration of the aeroengine rotor and collect the vibration data.

The structure of the simulation platform is shown in Figure 3. The simulation platform is mainly composed of direct current motor, transmission shaft, two rotors (simulation of double rotor engine), bracket and base. The self built simulation engine rotor vibration platform could set different rotor faults independently. The blind source separation algorithm, FDM algorithm and SVM algorithm were compiled by MATLAB software [15] to monitor the engine rotor fault.

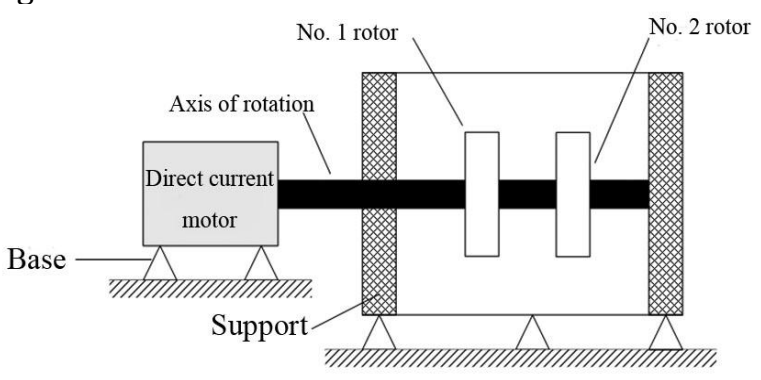

Figure 3: The structural diagram of self built simulation engine rotor vibration platform

\subsection{Experiment setup}

(1) An acceleration vibration sensor was fixed on the engine rotor case using a magnet.

(2) The self built analog engine rotor was started. The speed of the rotor was $550 \mathrm{r} / \mathrm{min}$, and three operating states were set respectively: normal rotation, No.1 rotor outer ring fault and No. 2 rotor outer ring fault. Then the vibration signals under three operating states were collected through the sensor: $10 \mathrm{~s}$ was taken as a sampling cycle, the sampling point of each cycle was set as 1024, and each state was sampled for 30 cycles. Two thirds of them were taken as the training set and one third was as the testing set.

(3) The training set was input into the SVM based on FDM and the SVM based on ICA-FDM respectively for training. The radial basis function was taken as $K\left(x_{i}, x_{j}\right)$ of the two SVMs. $C$ was set as 0.01 .

(4) After feature extraction, the vibration signals in the testing set were input into the trained SVM to obtain the recognition results.

\subsection{Experimental results}

In the simulation experiment, although the vibration signal of the engine rotor collected by the vibration sensor is periodic to some extent, the details are disorderly. It is difficult to see the difference between vibration signals of the rotor in different operating states as a whole. Limited to the space, this paper only shows the vibration signal of one cycle. As shown in Figure 4, it is difficult to see the obvious vibration characteristics. For the vibration signal features extracted by two methods, limited by space, only the vibration features extracted by FDM and ICA-FDM under the state of No. 2 rotor outer ring fault are shown. The vibration features extracted by FDM are shown in Figure 5.

It was seen from Figure 5 that the vibration features extracted by FDM contained fundamental frequency $f_{0}$, twofold frequency, threefold frequency, fourfold frequency and fivefold frequency of rotor speed. In addition to the prominent fundamental frequency, the fivefold frequency is also relatively prominent. The frequency corresponding to the fivefold frequency is very close to the theoretical frequency of $23.95 \mathrm{~Hz}$ when the rotor outer ring fails (the theoretical frequency of the rotor outer ring failure is related to the rotor platform, which can be calculated using the following formula:

$$
2 f_{c}=z f_{n}(1-d \cos \alpha / D)
$$

where $f_{c}$ is the theoretical frequency when rotor outer ring fails, $z$ is an influence factor, $f_{n}$ is the frequency of the axis of rotation, $d$ is the ball diameter, $D$ is the bearing raceway diameter, and $\alpha$ is contact angle), that is to say, features in Figure 4 reflect that the outer ring of the rotor has failure, but it is difficult to judge from Figure 4 which one has failure, No. 1 or No. 2.

The vibration features extracted by ICA-FDM are shown in Figure 5 and 6. In the method of ICA-FDM, blind source separation was carried out for the vibration signals, so the fault vibration features of No. 1 and 2 rotors were separated, which are respectively shown in Figure 6 and 7. It was seen from the figures that the frequency of $23.51 \mathrm{~Hz}$ was the most prominent in the vibration features of No. 1 rotor, and it was close to the theoretical frequency when the outer ring of rotor was in fault. 
Considering that the frequency distribution in the vibration features of No. 2 rotor, it was inferred that No. 1 rotor had outer ring fault.

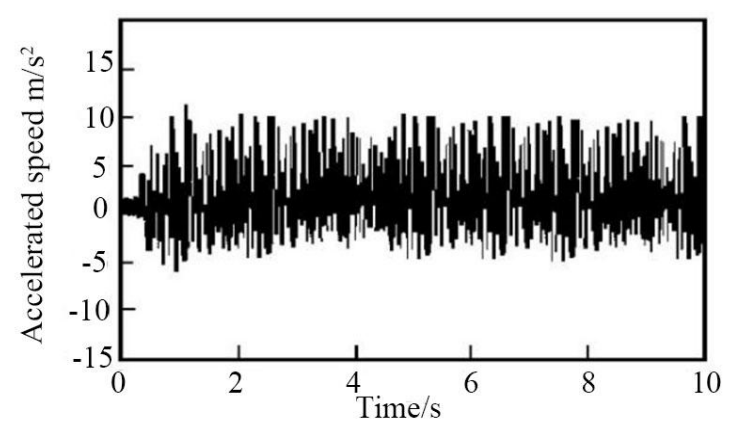

Figure 4: The vibration signal collected by the vibration sensor

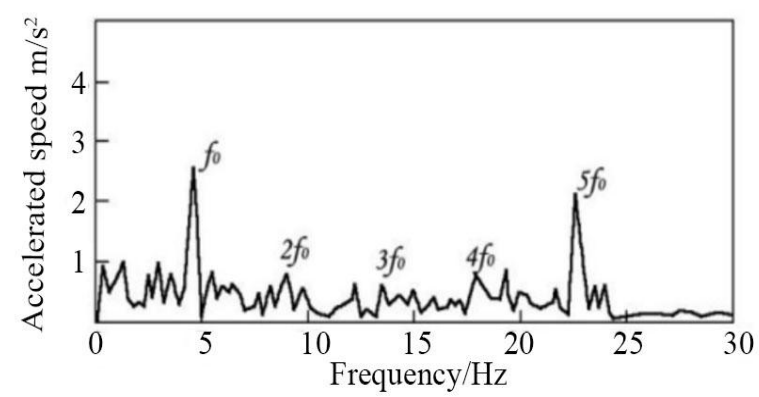

Figure 5: The vibration feature of rotor fault after extraction by FDM

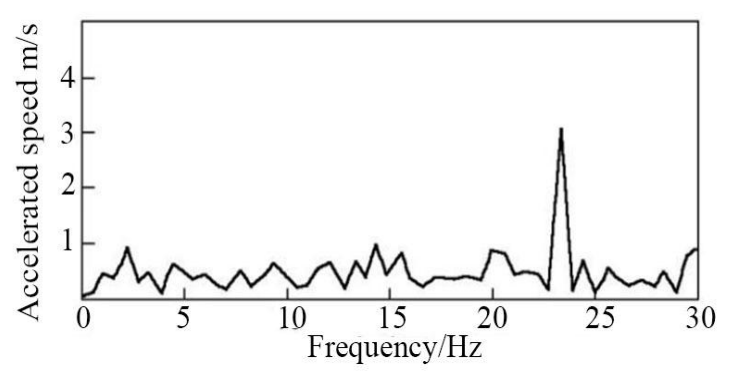

Figure 6: The vibration feature of No. 1 rotor after ICA-FDM separation

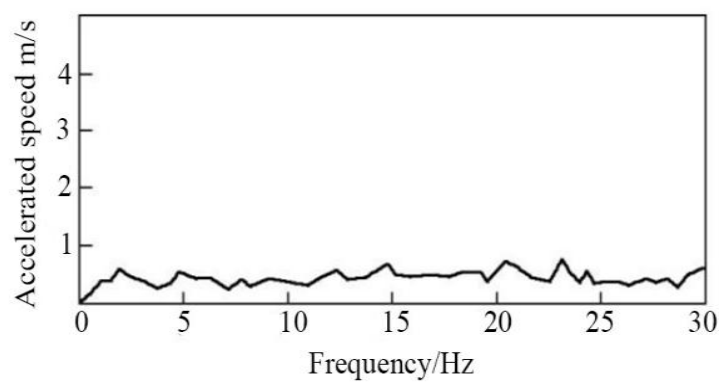

Figure 7: The vibration feature of No. 2 rotor after ICA-FDM separation

Figure 8 shows the vibration signal of one cycle collected by the vibration sensor from the self built rotor platform when No. 2 fails.
The signal had a rough vibration range, but the specific rules can not be seen directly by the naked eye. Compared with the vibration signal of No. 1 rotor in Figure 4, there was little difference. Figure 9 is the vibration feature after FDM extraction of the vibration signal in Figure 8. It was seen from Figure 9 that it includes the fundamental frequency, twofold frequency, threefold frequency, fourfold frequency and fivefold frequency of rotor speed. In addition to the prominent fundamental frequency, the fivefold frequency is also relatively prominent. The frequency corresponding to the fivefold frequency is very close to the theoretical frequency of $23.95 \mathrm{~Hz}$ when the rotor outer ring fails. The comparison of Figure 5 and 9 shows that two vibration features were very close; although the details of the nonfeature frequency were different, they were basically the same in the fundamental frequency and fold frequency. Considering the two vibration feature figures corresponding to the outer ring faults of different rotors, it was seen that it was difficult to distinguish which rotor had faults although FDM could obtain vibration features effectively. Figure 10 and 11 are the vibration features extracted from the vibration signal in Figure 8 by ICA-FDM. It was seen from Figure 8 that the frequency distribution in the vibration feature of No. 1 rotor was relatively stable, and there was no particularly prominent characteristic frequency; the frequency of $23.51 \mathrm{~Hz}$ in the vibration feature of No. 2 rotor was very prominent in the distribution, which was quite close to the vibration frequency when the outer ring of rotor failed. The comparison between Figure 6 and 7 and between Figure 10 and 11 shows that the vibration feature obtained by ICA-FDM was very similar to the rotor with outer ring fault and which rotor has fault can be very intuitively determined through the blind source separation based on ICA.

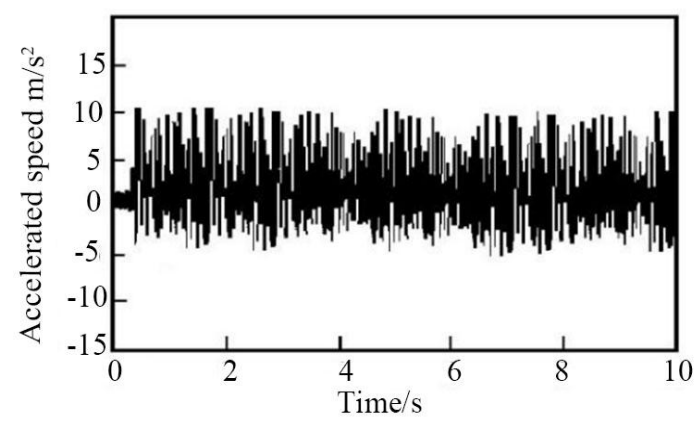

Figure 8: Vibration signal collected by vibration sensor 


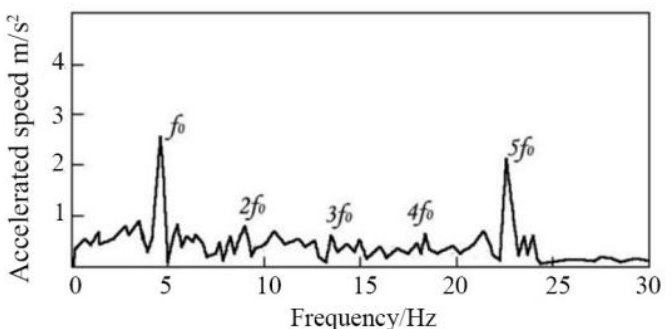

Figure 9: Vibration feature extracted by FDM

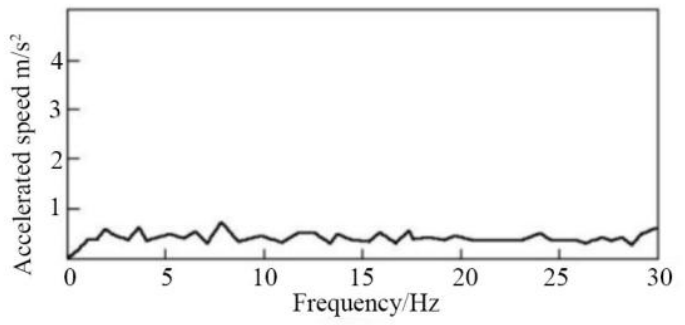

Figure 10: Vibration feature of No. 1 rotor extracted by ICA-FDM

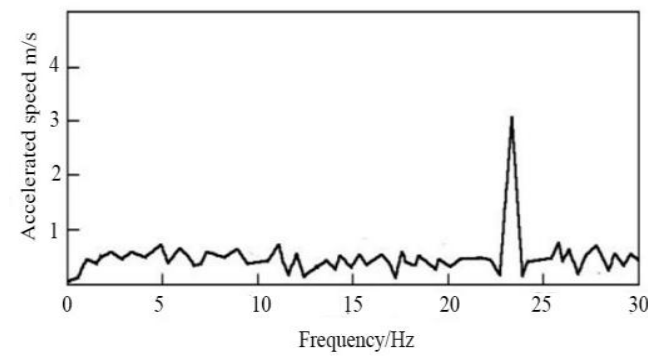

Figure 11: Vibration feature of No. 2 rotor extracted by ICA-FDM

It was seen from the above comparison that although the vibration features extracted by FDM could effectively separate the fundamental frequency and multiple frequency from the vibration signal, so as to effectively determine whether the rotor was in fault, it is difficult to determine which rotor was in fault; the vibration features extracted by ICA-FDM could also effectively determine the rotor fault frequency and restore the vibration source after blind source separation, so as to judge the rotor in fault easily.

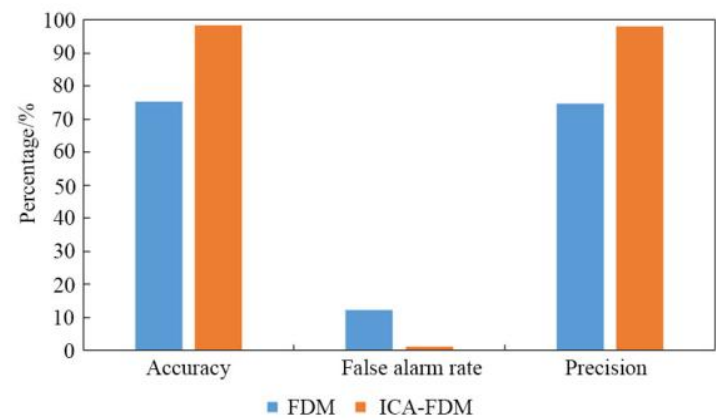

Figure 12: The performance of SVM for recognizing single rotor outer ring fault under two feature extraction methods
After the two feature extraction methods were applied to obtain the vibration features of the engine rotor, SVM was trained, and then SVM was tested under the two feature extraction methods using the testing set. The results are shown in Figure 12. The accuracy of SVM based on FDM for recognition of single rotor outer ring fault was $75.3 \%$, the false alarm rate was $12.4 \%$, and the recognition precision was 74.8\%; the accuracy of SVM based on ICA-FDM for recognition of single rotor outer ring fault was $98.3 \%$, the false alarm rate was $1.2 \%$, and the recognition precision was $98.1 \%$. It was seen intuitively from Figure 12 that the accuracy and precision of SVM which applied ICA-FDM to extract vibration features for engine rotor fault vibration recognition were significantly higher than those using FDM, while the false alarm rate of the SVM based on ICA-FDM was higher. The reason is as follows. Although the vibration source of the aliasing signal was separated by FDM, most of the separated features were concentrated on the fundamental frequency and multiple frequency, and although these features were obtained by decomposition, they were still based on the overall vibration features, so it was impossible to determine which rotor the features in the fundamental frequency and multiple frequency came from; when ICA-FDM extracted the vibration features, it first separated the blind source and restored the vibration signals of each vibration source as much as possible, which made the extracted vibration features closer to the actual features, and moreover it can determine which rotor the fault vibration came from.

\section{Conclusions}

In this study, Fourier separation method and SVM were combined to realize the vibration monitoring of aeroengine rotor fault, and ICA was used to improve the method used for extracting vibration features. After that, the two monitoring methods before and after the improvement were compared by experiments. The results are as follows: (1) the vibration signals of rotor under different operating states obtained by sensors were similar, and the vibration feature extracted by FDM contained the basic frequency and multiple frequency which reflected the fault features, but the vibration source of the fault vibration could not be distinguished; the feature extracted by ICA-FDM contained the feature 
frequency of fault, and the fault vibration source could be distinguished; (2) after training, the recognition accuracy of SVM based on FDM was $75.3 \%$, the recognition false alarm rate was $12.4 \%$, the recognition precision was $74.8 \%$; the recognition accuracy of SVM based on ICA-FDM was 98.3\%, the recognition false alarm rate was $1.2 \%$, and the recognition precision was $98.1 \%$.

\section{References}

[1] Chen G. "Simulation of casing vibration resulting from blade-casing rubbing and its verifications," Journal of Sound and Vibration, 2015, 361:190-209.

[2] Zhuo M. "Analysis of Rotor Thermal Bow and Vibration Response in Gas Turbine," Journal of Mechanical Engineering, 2017, 53(3):57.

[3] Zhang J, Cao Y, Li Y. "Aero-engine Health Monitoring Method Based on Optimized Grey Target Theory," Journal of Vibration Measurement \& Diagnosis, 2018, 38(2):228233.

[4] Ma X X, Ma H, Zeng J, Piao Y. "Rubbing-Induced Vibration Response Analysis of Dual-RotorCasing System," Transactions of Nanjing University of Aeronautics and Astronautics, 2018, 35(1).

[5] Wang Y F, Zhu J, Teng G R, Liang EB. "11/2dimension spectrum analysis on early cracked fault characters of aero engine rotors," Journal of Vibration \& Shock, 2015, 34(1):88-93 and 134.

[6] Ding F, Li X, Han S. "Application of EEMD and NRS in turboprop engine rotor fault diagnosis," Journal of Aerospace Power, 2018, 33(6):14231431.

[7] $\mathrm{Yu}$ L, Qu J, Gao F. "Missing vibration data reconstruction using compressed sensing based on over-complete dictionary," Systems Engineering \& Electronics, 2017, 39(8):18711877.
[8] Hazra M, Kumar J, Singh S. "Failure of Locking Wires of an Aeroengine Component: Attributed Primarily to Over-twisting and Secondarily to Engine Vibration and Improper Material Selection," Journal of Failure Analysis and Prevention, 2019, (2).

[9] Rota-Rodrigo S, Lopez-Aldaba A, Perez-Herrera R A, Lopez Bautista M D C, Esteban Ó, LopezAmo M. "Simultaneous Measurement of Humidity and Vibration Based on a Microwire Sensor System Using Fast Fourier Transform Technique," Journal of Lightwave Technology, 2016, 34(19):4525-4530.

[10] Tollis G, Chiariotti P, Martarelli M. "Rolling Bearing Diagnostics by Means of EMD-Based Independent Component Analysis on Vibration and Acoustic Data," 2017, 9:293-300.

[11] Yao J, Xiang Y, Qian S, Wang S, Wu S. "Noise source identification of diesel engine based on variational mode decomposition and robust independent component analysis," 2017, 116:184-194.

[12] Yang Y, Liu Y X, Liu Z B. "Prediction of Yueqin acoustic quality based on soundboard vibration performance using support vector machine," Journal of Wood Science, 2016, 63(1):1-8.

[13] Ma X J, Ren S H, Zuo H F. "Prediction method of aero-engine life on wing based on LS-SVM algorithm and performance reliability," Journal of Traffic \& Transportation Engineering, 2015, 15.

[14] Wang S L, Ding X H, Zhu D Y. "Measurement Uncertainty Evaluation in Whiplash Test Model via Neural Network and Support Vector Machine-Based Monte Carlo Method," Measurement, 2018, 119.

[15] Liu Y, Wang Q, Liu K, Zhang Y M. "Micro Milling Cutter Breakage Detection Based on Wavelet Singularity and Support Vector Machine," Journal of Northeastern University, 2017, 38(10):1426-1430. 